\title{
Correction to: In silico molecular docking and dynamic simulation of eugenol compounds against breast cancer
}

\author{
Hezha O. Rasul ${ }^{1}$ (D) Bakhtyar K. Aziz ${ }^{2}$. Dlzar D. Ghafour ${ }^{3,4} \cdot$ Arif Kivrak $^{5}$ \\ Published online: 5 March 2022 \\ ๑) Springer-Verlag GmbH Germany, part of Springer Nature 2022
}

\section{Journal of Molecular Modeling (2022) 28: 17 https://doi.org/10.1007/s00894-021-05010-w}

This corrects the article "In silico molecular docking and dynamic simulation of eugenol compounds against breast cancer" in volume (28) 17.

In the published article "In silico molecular docking and dynamic simulation of eugenol compounds against breast cancer" J Mol Model 28, 17 (2022), https://doi.org/10.1007/s00894021-05010-w" two affiliations were published incorrectly. The authors apologize for any inconvenience that it may have caused.

Hezha O. Rasul ${ }^{1}$, Bakhtyar K. Aziz ${ }^{1}$, Dlzar D. Ghafour ${ }^{2,3}$, Arif Kivrak $^{4}$

${ }^{1}$ College of Medicals and Applied Sciences, Charmo University, Peshawa Street, Chamchamal, 46,023, Sulaimani, Iraq

The original article can be found online at https://doi.org/10.1007/ s00894-021-05010-w.

Hezha O. Rasul

hezha.rasul@charmouniversity.org

1 Department of Pharmaceutical Chemistry, College of Medicals and Applied Sciences, Charmo University, Peshawa Street, Chamchamal, 46023 Sulaimani, Iraq

2 Department of Nanoscience and Applied Chemistry, College of Medicals and Applied Sciences, Charmo University, Peshawa Street, Chamchamal, 46023 Sulaimani, Iraq

3 Department of Medical Laboratory Science, College of Science, Komar University of Science and Technology, 46001 Sulaimani, Iraq

4 Department of Chemistry, College of Science, University of Sulaimani, 46001 Sulaimani, Iraq

5 Department of Chemistry, Faculty of Sciences and Arts, Eskisehir Osmangazi University, Eskişehir 26040, Turkey
${ }^{2}$ College of Science, Department of Medical Laboratory Science, Komar University of Science and Technology, 46,001, Sulaimani, Iraq

${ }^{3}$ College of Science, Chemistry Department, University of Sulaimani, 46,001, Sulaimani, Iraq

${ }^{4}$ Eskisehir Osmangazi University, Faculty of Sciences and Arts, Department of Chemistry, Eskişehir, 26,040, Turkey Email (corresponding): hezha.rasul@charmouniversity.org

The two affiliations should be corrected as follows:

Hezha O. Rasul ${ }^{1}$, Bakhtyar K. Aziz², Dlzar D. Ghafour ${ }^{3,4}$, Arif Kivrak ${ }^{5}$

${ }^{1}$ Department of Pharmaceutical Chemistry, College of Medicals and Applied Sciences, Charmo University, Peshawa Street, Chamchamal, 46,023, Sulaimani, Iraq

${ }^{2}$ Department of Nanoscience and Applied Chemistry, College of Medicals and Applied Sciences, Charmo University, Peshawa Street, Chamchamal, 46,023, Sulaimani, Iraq ${ }^{3}$ Department of Medical Laboratory Science, College of Science, Komar University of Science and Technology, 46,001, Sulaimani, Iraq

${ }^{4}$ Department of Chemistry, College of Science, University of Sulaimani, 46,001, Sulaimani, Iraq

${ }^{5}$ Department of Chemistry, Faculty of Sciences and Arts, Eskisehir Osmangazi University, Eskişehir, 26,040, Turkey Email (corresponding): hezha.rasul@charmouniversity.org

The original article has been corrected.

Publisher's Note Springer Nature remains neutral with regard to jurisdictional claims in published maps and institutional affiliations. 